\title{
Computation of Breast Ptosis from 3D Scans of Torso
}

\author{
Danni Li ${ }^{1}$ (dannylee2012@gmail.com), Lijuan Zhao² (ruthzhao08@gmail.com), Gregory P. Reece ${ }^{3}$ \\ (greece@mdanderson.org), Melissa A. Crosby ${ }^{3}$ (macrosby@mdanderson.org), Michelle C. Fingeret ${ }^{3,4}$ \\ (mcfinger@mdanderson.org), and Fatima A. Merchant ${ }^{* 1,2,5}$ (fmerchant@uh.edu) \\ ${ }^{1}$ Department of Electrical and Computer Engineering, University of Houston, Houston, TX \\ ${ }^{2}$ Department of Computer Science, University of Houston, Houston, TX \\ ${ }^{3}$ Department of Plastic Surgery, The University of Texas MD Anderson Cancer Center, Houston, TX \\ ${ }^{4}$ Department of Behavioral Science, The University of Texas MD Anderson Cancer Center, Houston, TX \\ ${ }^{5}$ Department of Engineering Technology, University of Houston, Houston, TX \\ Corresponding Author \\ http://dx.doi.org/10.15221/13.098
}

Ptosis is an important morphological parameter for characterizing breast aesthetics and is frequently assessed before breast surgery. It refers to the extent to which the nipple is lower than the inframammary fold (the contour along which the inferior part of the breast attaches to the chest wall). Current clinical assessment of ptosis involves qualitative visualization by observers, which is subject to inter- and intra-observer variability. Alternatively, ptosis can be measured anthropometrically directly by manual measurements from the patient or indirectly from manual or computerized measurements on clinical photographs. Although functional, these methods are subject to operator bias and can be practically limiting. As stereophotography is now finding its niche in clinical breast surgery, in this study we investigated and evaluated the utility of three-dimensional (3D) features such as surface curvature, coronal projection and surface normal for the assessment of breast ptosis using 3D scans of the torso. Experimental results suggest that 3D features are successful for objectively categorizing breast ptosis with high accuracy and precision.

Keywords: 3D image, Breast surgery, Ptosis, Gaussian curvature, Coronal projection, Classification

\section{Introduction}

Ptosis is a measurement clinically used for characterizing breast morphology that estimates the amount of sagging or drooping of the breast. It refers to the extent by which the nipple is lower than the inframammary fold (IMF), i.e., the lower breast contour along which the inferior part of the breast attaches to the chest wall. Currently, measures characterizing ptosis are determined by (1) qualitative subjective assessment by human observers, (2) direct physical measurements (anthropometry), (3) computer aided measurements on clinical photographs (photogrammetry), and (4) computer aided measurements using three-dimensional (3D) images (stereophotogrammetry).

Subjective assessment of breast aesthetics is highly influenced by the observers' experience and may be biased based on his/her visual perception of breast aesthetics. This assessment is typically based on vaguely defined rating scales that are inherently subjective and qualitative. Substantial studies have reported low intra- and inter- observer agreement and reliability, primarily due to the lack of consistency in the manual perception and interpretation of aesthetic outcomes [1-3].

Anthropometry is a measurement performed directly on the patient's body using a measuring tape. Occasionally, measurement of fundamental parameters such as distances along the contoured surface of the breast can be imprecise due to the inherent mobility of the subcutaneous glandular tissues. Despite being a useful approach for quantifying breast aesthetics in clinical practice, there are several pragmatic limitations that make it impractical when evaluating a large group of patients in clinical studies.

Photogrammetry is an alternative allowing indirect anthropometry on two-dimensional (2D) clinical photographs. In photogrammetry, digital images are typically displayed on a computer monitor, or conventional photographs are shown to observers for manually marking measurements. Photogrammetry is relatively more feasible and easy to implement since most medical institutions routinely take photographs for documentation purposes. However, photogrammetry has its draw- backs too. It cannot capture the 3D nature of the human torso. To get a complete view of the torso, a set of multiple photographs with the patient positioned at different angles have to be taken. Furthermore, accurate anatomic landmarks that are critical to obtaining reproducible assessment of aesthetic outcomes by photogrammetry may not be visible from 2D photographs. Some studies have also 
reported substantial observers' deviation for assessment from photographs, due to the lack of consistent guidelines for standard photography $[4,5]$.

Stereophotogrammetry, which involves measurements on 3D scans of torso, is being evaluated as an alternative method to assess breast aesthetics. 3D digital photography systems are capable of noninvasively generating precise images at high speeds. A single 3D image yields more information on breast appearance than multiple conventional 2D photographs. One 3D scan of the torso can be viewed from several different angles, which is impossible in 2D photogrammetry. They also enable objective determination of properties such as contour distance, surface area, volume, and surface curvature, which are not available from $2 \mathrm{D}$ images [6-8]. Thus, 3D imaging has tremendous potential for analysis of breast appearance.

Previous studies on breast ptosis measurements were all based either on subjective ratings, direct anthropometry or 2D photogrammetry, thereby affecting the analysis that can be performed. Ptosis ratings involve identification of fiducial points (such as the nipple and the inframammary fold) on patients. However, the inframammary fold is often difficult to identify when done by photogrammetry and stereophotography because it is vaguely defined and hard to distinguish [12]. Currently, 3D stereophotogrammetry is finding its niche in surgical planning, patient education and evaluation of surgical outcomes. 3D features can be potentially explored for quantitative assessment of ptosis, which has not been done in previous studies. In this paper, we present an approach of measuring ptosis that excludes the identification of the inframammary fold using 3D scans of torso, and would provide robust performance and accurate results.

\section{Related Work}

\subsection{Qualitative Classifications of Breast Ptosis}

Plastic surgeons use a classification system to categorize the degree of breast sagging, or ptosis. This classification system also helps surgeons determine what treatment option is the best for an individual based on the level of her breast ptosis. Ptosis is clinically defined using Regnault's (1976) [9] classification scheme (Figure 1), wherein Grade 0 (Normal breast) is defined as a normal breast that has the nipple and parenchyma (glandular tissue and fat which compose the breast) sitting above the IMF. In Grade 1 (Minor ptosis), the nipple lies at the level of the IMF and above the lowest contour of the breast. In Grade 2 (Moderate ptosis), the breast exhibits sagging in which the nipple lies below the level of the IMF but remains above the lowest contour of the breast, and in Grade 3 (Major ptosis), the breast exhibits severe sagging in which the nipple lies well below the IMF and lies at the inferior contour of the breast.
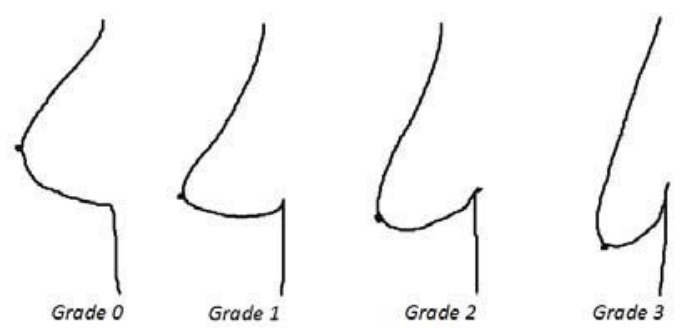

Figure 1: Regnault's classification of ptosis.

Additionally, a variety of other classification schemes exist for determining the degree of ptosis, including those described by Lewis (1983) [13], Brink (1990) [14] and (1993) [15], LaTrenta and Hoffman (1994) [10], Kirwan (2002) [16], and de la Torre and Vasconez (2007) [17]. Classification of ptosis can be used to help determine the type of surgery, such as for the determination of whether to augment the breast, perform a mastopexy or do a breast reduction. Ptosis assessments are also used by physicians when discussing with the patient the pros and cons of different surgical procedures [18].

\subsection{Quantitative Classification of Breast Ptosis}

In 1994, LaTrenta and Hoffman [10] added a quantitative measurement for the Regnault's classification of ptosis based on the vertical distance of the nipple to the IMF. They quantified the classification using distance in centimeter metrics as follows.

First degree or minor ptosis: nipple position lies within $1 \mathrm{~cm}$ of the level of the IMF. 
Second degree or moderate ptosis: nipple position lies 1-3 $\mathrm{cm}$ below the IMF.

Third degree or severe ptosis: nipple position lies greater than $3 \mathrm{~cm}$ below the level of the IMF and below the lower contour of the breast and skin envelope.

Pseudoptosis: nipple position lies at or above the level of the IMF with a loose "saggy" skin envelope, giving the impression of true ptosis.

In addition, Kirwan [16] proposed a new quantitative measurement for ptosis wherein 6 stages of breast ptosis covering a $5 \mathrm{~cm}$ distance are defined. Stages $A$ to $F$ progress in $1 \mathrm{~cm}$ increments as follows: (A) nipple position $2 \mathrm{~cm}$ above the IMF, (B) nipple position $1 \mathrm{~cm}$ above the IMF, (C) nipple position even with IMF, (D) nipple position $1 \mathrm{~cm}$ below the IMF, (E) nipple position $2 \mathrm{~cm}$ below the IMF, and (F) nipple position greater than $2 \mathrm{~cm}$ below the IMF.

\subsection{Objective Measurements of Ptosis using 2D Photogrammetry}

Kim et al. [11] proposed an objective, quantitative measurement of breast ptosis based on the ratio of distances between fiducial points, such as nipple, sternal notch, lateral terminus, and lowest visible point, manually identified in digitized/digital images of oblique and lateral preoperative photographs. However, the automatic identification of fiducial points can be challenging to locate and manual interventions must be involved. Furthermore, outcomes of distance ratios, which scale from 0 to 1 need to be accurately mapped on to the 4-point scale by Regnault to allow interpretation of the outcome in terms of the ptosis grade. The paper employed a simple linear regression approach to transform the distance ratios to subjective scales, based on two clinical groups of 52 patients and 10 patients, respectively. However, the distribution of the measurements in either group did not illustrate strong linear relationships with the Regnault's 4-point scale ( $R^{2}$ values of 0.4808 and 0.5793$)$.

\section{Data Acquisition}

\subsection{Imaging System}

The 3D images used in this study were captured using two stereophotogrammetric systems, namely, the DSP800 and 3dMDTorso systems manufactured by 3Q Technologies Inc., Atlanta, GA. The latest version, the 3dMDTorso, has improved accuracy enabling capture of 3D data clouds of 75,000 points, whereas the older system, the DSP800, allows capture of data clouds consisting of 15,000 points. Each reconstructed surface image consists of a 3D point cloud, i.e., $x, y, z$ coordinates, and the corresponding $2 \mathrm{D}$ color map. Only the frontal portion of the torso is imaged resulting in a surface mesh, which excludes the back region.

\subsection{Study Sample}

Women scheduled to undergo mastectomy and breast reconstruction were recruited at The University of Texas MD Anderson Cancer Center under a protocol approved by the institutional review board. For this study, a data set consisting of torso images of 41 women who had either one or both nipple(s) visible were selected. In addition, images from 5 commissioned participants were included in the data set. The 41 patients ranged in age from 34 to 66 years $(48.5 \pm 9.4)$ with body mass index (BMI) in the range of 20.3 to $41 \mathrm{~kg} / \mathrm{m}^{2} \quad(30.2 \pm 6.2)$. Of the 41 patients, five were Hispanic/Latino and thirty-four were not Hispanic/Latino. Thirty-four patients were white and five were African American. Race, ethnicity, age, and BMI information was not available for two patients and the five commissioned female participants. From the total of 46 torso images, 64 cropped images (see section 4.1 below) consisting of the right or left breast were used for the computation of ptosis for each individual breast.

\section{Methodology}

\subsection{Image Cropping}

As a first step, the torso image was cropped to remove the neck, arms, legs and abdomen, leaving only the upper portion of the torso encompassing the breast as the region of interest (ROI). As shown in Figure 2, the ROI was defined from the vertical level of sternal notch down to the lowest visible point of the breast. Horizontally, each breast was cropped individually from the medial axis to the lateral point. The reason for cropping the torso image into individual breast regions is that the ptosis grade was determined individually for each breast, since a patient may either have asymmetric breasts, i.e., the two breasts may have different ptosis grades, or only one breast is intact due to whole or partial mastectomy. 
Furthermore, each breast was divided into four quadrants with the nipple at the origin to capture any spatial morphological differences across the four ptosis grades. The four quadrants were named as $a$, $b, c$, and $d$ clockwise as follows: the upper inner quadrant $a$, the upper outer quadrant $b$, the lower outer quadrant $c$, and the lower inner quadrant $d$ (see Figure $2 \mathrm{~d}$ ). As the ptosis grade increases, the nipple moves downward, and the shape of the breast within each quadrant as well as the distribution of points in each quadrant changes across the four grades.

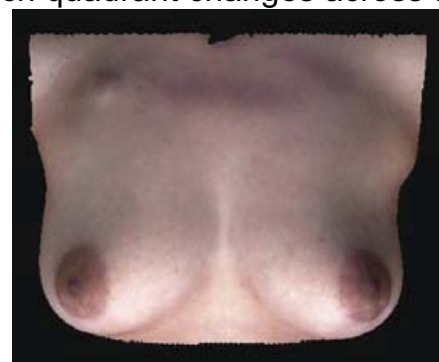

(a)

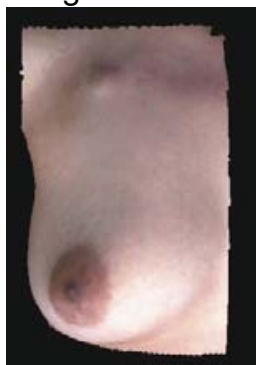

(b)

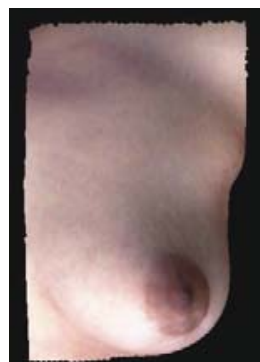

(c)

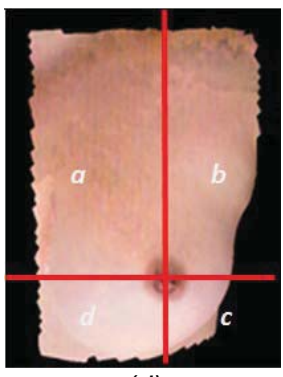

(d)

Figure 2. Image of torso (from the sternal notch to just below the IMF) with the neck and arms cropped.

(a) Cropped image with both breasts (b) Cropped image of the right breast (c) Cropped image of the left breast (d) Each breast ROI was divided into 4 quadrants.

\subsection{Curvature Analysis}

Surface curvature analysis can be used to highlight the shape of the underlying 3D surface, thus it plays an important role in ptosis classification. To calculate Gaussian curvature, we used a toolbox developed by Gabriel Peyre [19] based on the algorithms proposed by Cohen-Steiner et al. [20,21]. We employed a pseudo-color visualization method for viewing the Gaussian curvature of the 3D mesh. Figure 3 shows the color-mapped Gaussian curvature for the 3D image in Figure 2. The color red represents elliptic regions, blue represents hyperbolic regions, and green represents regions that are nearly planar or cylindrical.

An ideal breast without ptosis is relatively symmetrical across the superior half and inferior half, whereas sagging of the breast gland with increasing ptosis results in a non-symmetric breast shape and consequent asymmetry in curvature is observed across the upper and lower poles of the breast. When the ptosis grade increases, the underlying gland droops, leading to a larger area that is flatter (low curvature values) being observed within the regions of quadrants $a$ and $b$. This indicates an increase in the number of points around curvature value equal to zero within these quadrants.

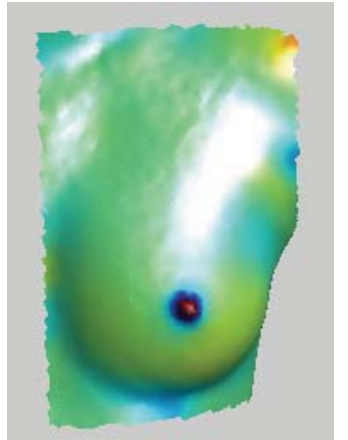

(a) Grade0

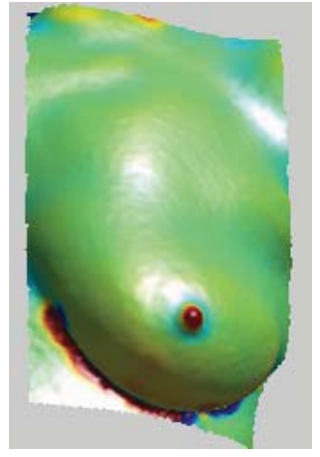

(b) Grade 1

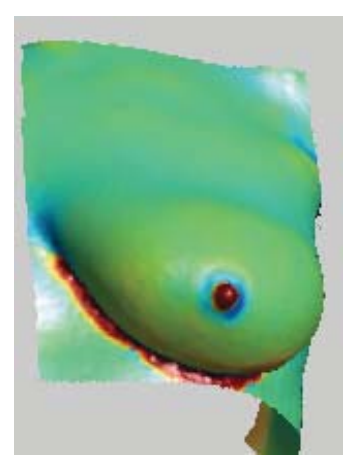

(c) Grade 2

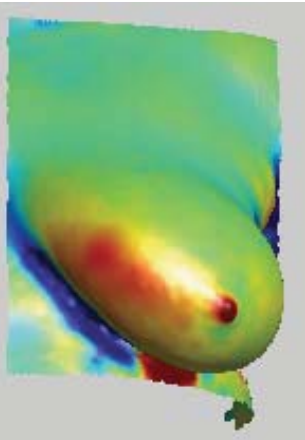

(d) Grade 3

Figure 3: Representative Gaussian curvature plots of breasts from the 4 ptosis grades.

For inferior quadrants $c$ and $d$, a reversal in the curvature values is observed. When the ptosis grade increases, the relative area of the lower two quadrants becomes smaller, and larger curvature values are apparent in the region below the IMF. Typically, a large portion of the breast exhibits a convex shape, in other words positive curvature values are observed in the nipple-areola area, whereas below the IMF, the concave shape of the crease introduces negative curvature values. Thus over the inferior pole of the breast a smaller number of points with lower curvature values are observed. Figure 3 shows the curvature map of four left breasts from grade $0,1,2$, and 3 . 
In order to evaluate the feasibility of Gaussian curvature as a feature for classification, we generated histograms to visualize the distribution of curvature values in each of the 4 quadrants. Since the curvature values are very small and greater than $95 \%$ of the values are located within the range $[-6 \times$ $10^{-4}, 6 \times 10^{-4}$ ], we divided the range into 40 bins, with each bin holding a range of $1.5 \times 10^{-5}$, except that the first bin and the last bin included Gaussian curvatures smaller than $-6 \times 10^{-4}$ and greater than $6 \times$ $10^{-4}$, respectively. In order to standardize the size of the breast, the total number of points in each bin was normalized with respect to the total number of points in the ROI. Histograms of four quadrants were generated and concatenated in the order of $a, b, c$, and $d$. Finally, a histogram template for every grade was generated by taking the average of the histograms for all breasts within the specific grade, as shown in Figure 4.

Figure 4 shows the Gaussian curvature histogram templates for grade 0, 1, 2, and 3, respectively. Quadrant $a, b, c$, and $d$ are represented in bins 1-40,41-80, $8-120$, and 121-160, respectively as shown in Table 4.1. Compared with grade 0 and grade 1 , it is clear that the number of points acquired around curvature value zero (bins 19-20 and 69-70) are larger, indicating a flatter area. For quadrants $c$ and $d$, the higher ptosis grade includes more low curvature values because of the drooping of the breast.

\subsection{Projection Analysis}

Coronal projection analysis is the second method, which was evaluated in order to incorporate the effect of breast shape on ptosis grade. A coronal plane is a vertical plane that divides the breast into front and back sections. Coronal projection analysis includes the computation of the total number of points located on the surface mesh, between every two subsequent coronal planes, as shown is Figure 5.
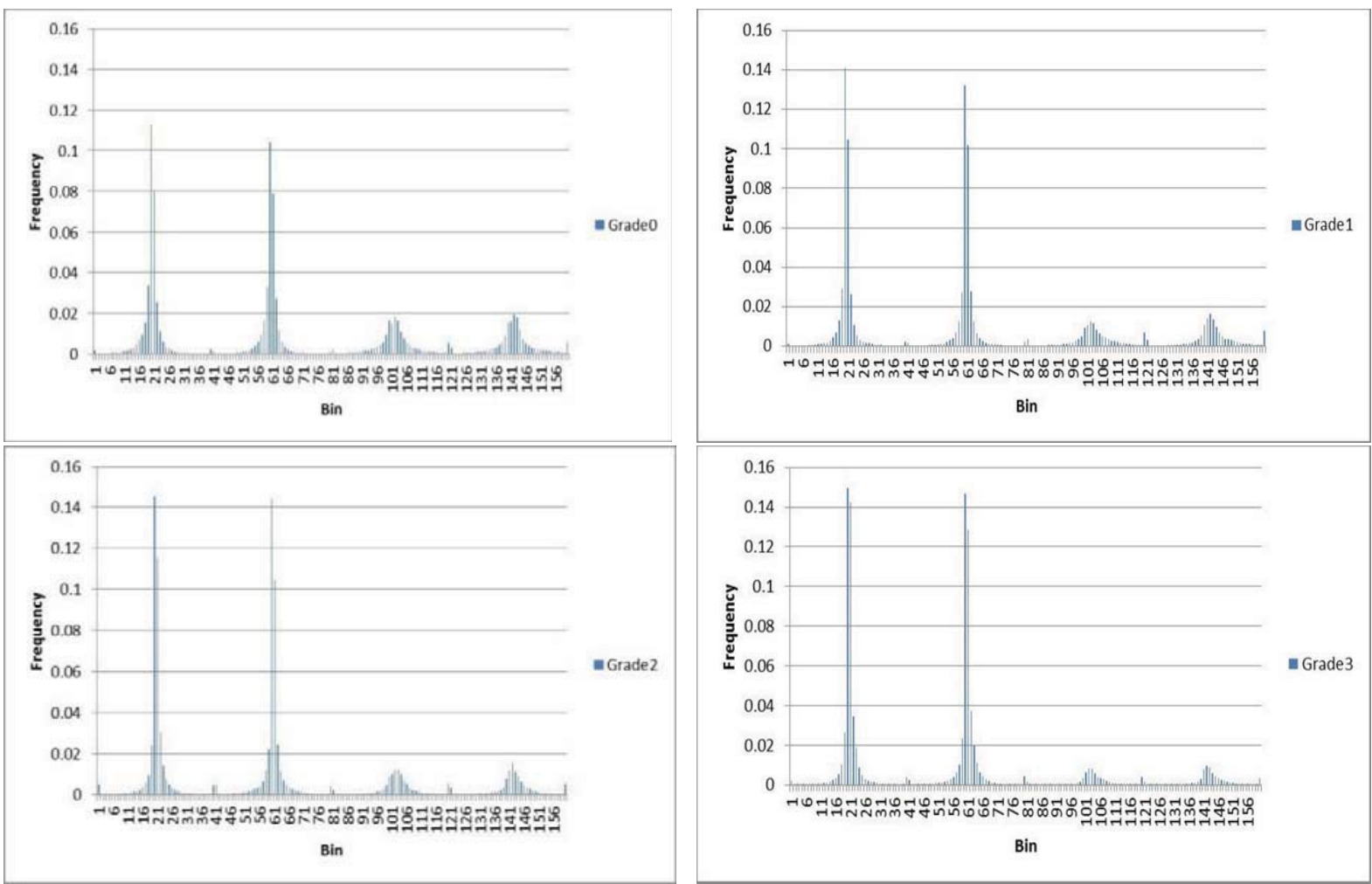

Figure 4. Histogram templates of concatenated Gaussian curvature histograms including each of the four quadrants (a-d) for Grade 0, Grade 1, Grade 2 and Grade 3. 


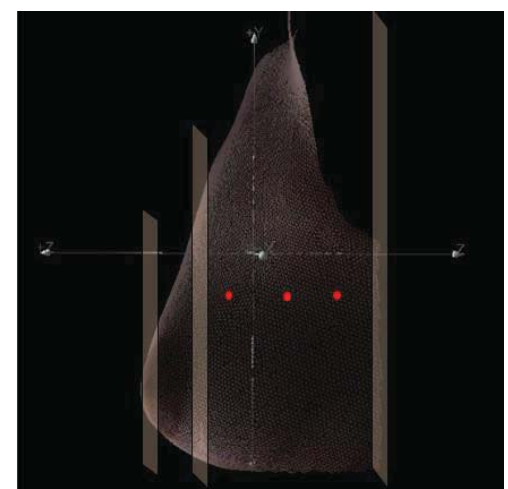

Figure 5: Coronal planes spaced at equal intervals placed depth-wise along the breast.

As mentioned before, each breast was divided into 4 quadrants according to the nipple position, and the same set of coronal planes were applied to all the 4 quadrants. We projected several coronal planes spaced at equal intervals to cut the breast into sections along the Z-axis. The depth of the breast was normalized along the Z-axis. The number of points located on the surface mesh on or between every two sequential planes were counted and normalized by the total number of points in the ROI.

Because the point density for each subject is the same within each ROI, we reasoned that the distribution of points on the torso represents the morphology of the breast. A coronal plane projection plot for each of the four grades is shown in Figure 6 to illustrate the idea. Contours shown in Figure 6 display points located on coronal planes with an interval of $6 \mathrm{~mm}$. It is important to note that nipple may or may not locate at the largest Z-value especially for highly ptotic breasts. For grade 0 , which shows no ptosis, contours of superior and inferior parts, are circularly symmetric. As the ptosis grade increases, contours tend to be elliptical and nonsymmetric.

Nine coronal planes cut the Z-axis into ten bins, with each bin representing a width of 0.1 . Histograms for four quadrants were generated and concatenated in the order of $a, b, c$, and $d$. Histogram templates for each grade were generated by taking an average of all the breasts within each grade category. Figure 7 shows the coronal projection histogram templates of grade $0,1,2$, and 3. Quadrant $a$ is represented in bins 1-10, quadrant $b$ is represented in bins 11-20, quadrant $c$ is represented in bins 2130 , and quadrant $d$ is represented in bins $31-40$. Within each quadrant, low bin numbers represent points closer to the chest, while higher bin numbers represent points closer to the nipple. As ptosis increases, the nipple position moves downwards and the total number of points acquired in quadrants a and $b$ increases, while the total number of points in quadrants $c$ and $d$ decreases.

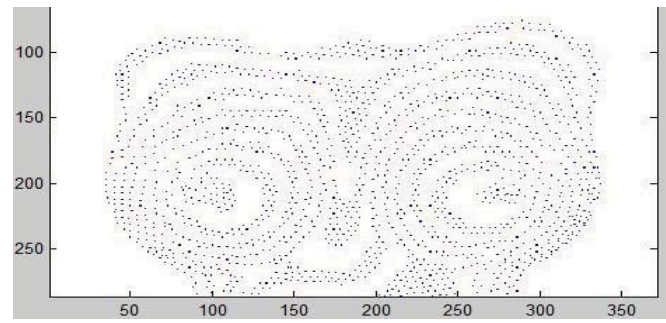

(a) Grade 0

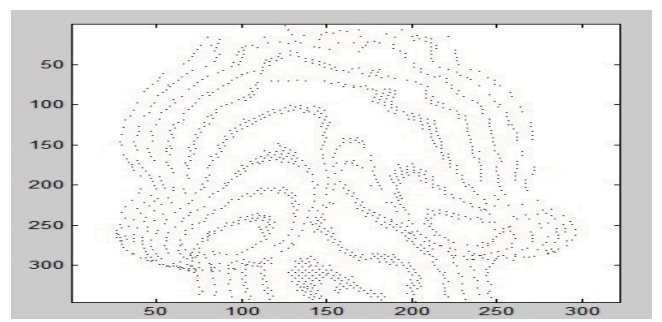

(c) Grade 2

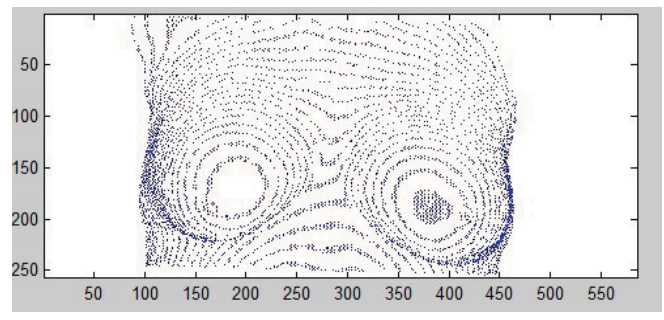

(b) Grade 1

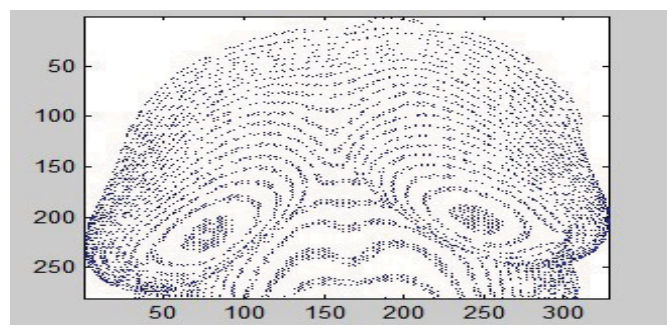

(d) Grade 3

Figure 6. Coronal projection plots for Grades 0, 1, 2, and 3. 

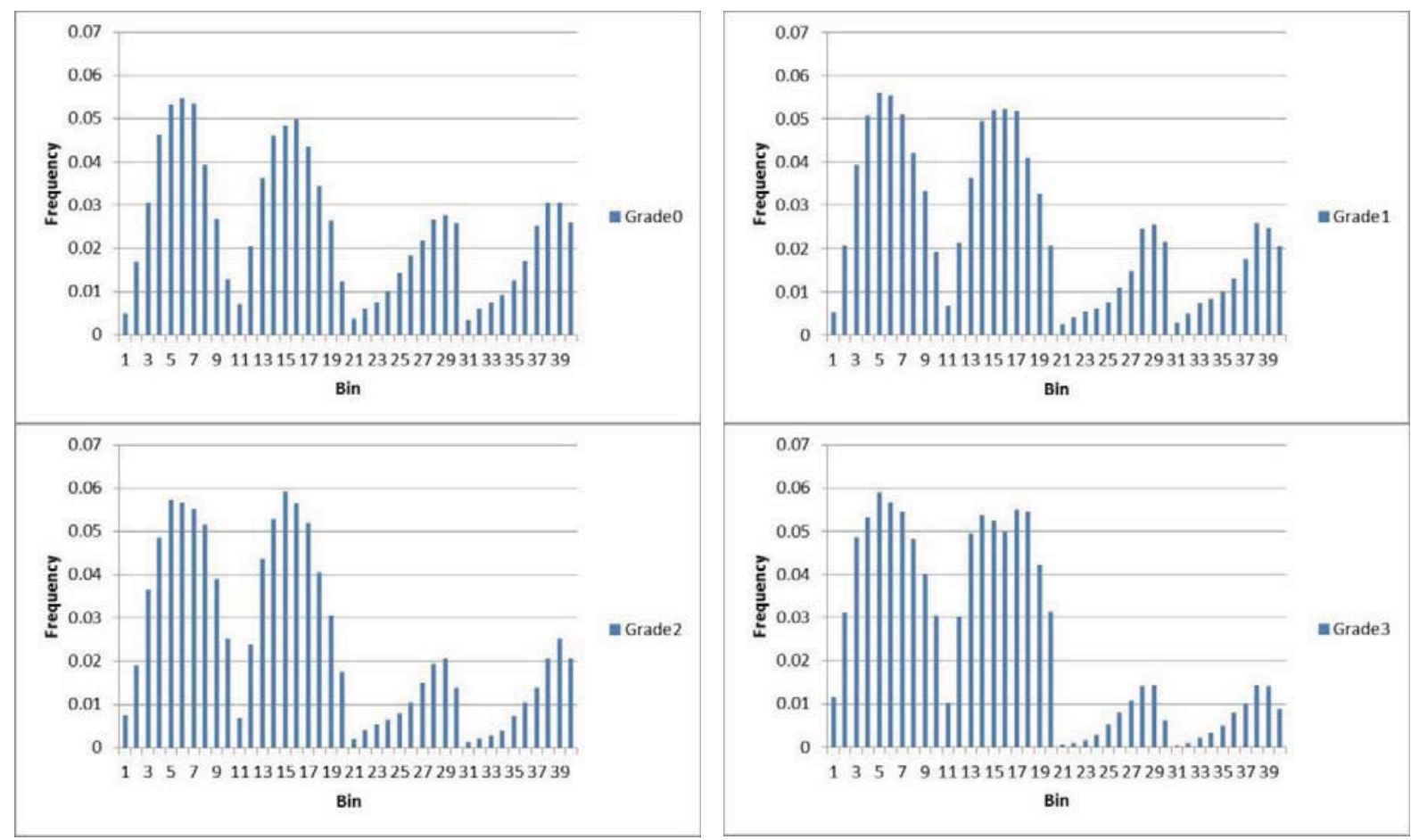

Figure 7: Histogram templates for coronal plane analysis for Grade 0, Grade 1, Grade 2 and Grade 3. Four quadrants are concatenated in the order: $a, b, c$, and $d$.

\subsection{Histogram Matching}

The similarity of the histogram template and the test case was computed by measuring the Bhattacharyya distance [64]. The Bhattacharyya measure can be used to compare the similarity between two histograms as follows. $H$ and $R$ represent two normalized histograms such that

And

$$
\sum \mathrm{H}_{\mathrm{i}}=1
$$

$$
\sum \mathrm{R}_{\mathrm{i}}=1
$$

If we let $H i$ be the histogram value in bin $i$ and $R i$, the histogram value in the same bin, the Bhattacharyya distance can be computed using the following equation

where

$$
\mathrm{d}(\mathrm{H}, \mathrm{R})=\sqrt{1-\frac{1}{\sqrt{\overline{\mathrm{H}} \overline{\mathrm{N}} \mathrm{N}^{2}}}} \sum_{\mathrm{i}} \sqrt{\mathrm{H}_{\mathrm{i}} \cdot \mathrm{R}_{\mathrm{i}}}
$$

$$
\overline{\mathrm{H}}=\frac{1}{\mathrm{~N}} \sum_{\mathrm{i}} \mathrm{H}_{\mathrm{i}}
$$

And $\mathrm{N}$ is the total number of bins in the histogram.

Bhattacharyya distances are computed to fall within the range of $[0,1] \cdot d(H, R)=0$ or closer to 0 means a better match, whereas $d(H, R)=1$ or closer to 1 indicates low similarity or a poor match. In order to compute the ptosis grade for a given test case, the Bhattacharyya distance between the test case's histogram and each of the four template histograms is computed, the smallest distance is noted and the grade of the corresponding template is set to be the predicted ptosis grade.

\section{Experiments and Results}

Cross-validation is a validation technique typically used to estimate the performance of a predictive model. For each round of cross-validation, the data set is partitioned into two complementary subsets, a training set and a test set. The training set is used to develop the methodology, while the test set is used to validate the analysis. Usually multiple rounds of cross-validation are performed on different partitions of the data set and the validation result is averaged over rounds. In leave-one-out crossvalidation (LOOCV), as its name suggests, the data set is partitioned into two subsets: one sample data 
in the test set and the remaining samples in the training set. It is repeated such that all samples are used once as a test set.

We performed LOOCV on the 64 breasts, including 16 breasts for each grade. For each test, we picked one breast out of the data set as the test case, and the remaining 63 breasts formed the training set. Template histograms were generated for each grade by taking average of all the individual histograms for each breast image in the training data set. The cross-validation analysis was repeated 64 times so that each subject image in the data set was used once as the validation data. Clinical ptosis rating by a plastic surgeon was used as the ground truth.

Each of the features, Gaussian curvature and coronal projection were tested individually and in combination. Our result shows that using Gaussian curvature and coronal projection independently has an average accuracy of $77 \%$ and $75 \%$, respectively, and the combined use of the two features has an accuracy of $75 \%$ (Table 1 ).

Table1. Averaged statistics for leave-one-out cross validation analysis.

\begin{tabular}{|c|c|c|c|}
\hline Statistic & Curvature & Coronal Projection & Combined \\
\hline Sensitivity & 0.5696 & 0.5054 & 0.5220 \\
\hline Specificity & 0.8483 & 0.8330 & 0.8319 \\
\hline Accuracy & 0.7734 & 0.7500 & 0.7500 \\
\hline
\end{tabular}

\section{Conclusion}

Development of an objective and quantitative method for measuring ptosis from 3D images is an important yet challenging task. Prior work mainly focused on subjective rating, anthropometry or $2 \mathrm{D}$ photogrammetry. In this study, we proposed a new approach for measuring breast ptosis using 3D torso scans. We explored unique 3D morphological features from stereophotogrammetry that surpass the need of predefining the IMF. We investigated Gaussian curvature, coronal projection, and their combination as features and built histogram models for each experiment. The results demonstrate that our new approach on 3D images yields good performance.

Future work in this study will include: 1) exploration of additional 3D features, 2) application of a multifeature discrimination statistical model for classification, and 3) automation of the assessment procedure, by incorporating computerized cropping and fiducial point detection to achieve robust and speedy prediction of breast ptosis. Automating the process of determining breast ptosis from 3D images should not only make the measurements of breast aesthetics more accurate and reliable, but also more practical for the busy medical profession.

\section{Acknowledgements}

This work was supported by a NIH grant 1R01CA143190-01A1. The patient data used in this study were generously provided by Steven J. Kronowitz, M.D., Patrick B. Garvey, M.D., Jesse C. Selber, M.D., Geoffrey L. Robb, M.D., Donald P. Baumann, M.D., Charles E. Butler, M.D., Roman J. Skoracki, M.D., Scott D. Oates, M.D., Matthew M. Hanasono, M.D., Alexander Nguyen, M.D., Charles E. Butler, M.D., David M. Adelman, M.D., Melissa A. Crosby, M.D., Gregory P. Reece, M.D., Mark Clemens, M.D., Edward Chang, M.D., Mark T. Villa, M.D., Elisabeth K. Beahm, M.D., and Justin M. Sacks, M.D. of the Department of Plastic Surgery at The University of Texas MD Anderson Cancer Center.

\section{Reference}

1. R. D. Pezner, J. A. Lipsett, N. L. Vora, and K. R. Desai, "Limited usefulness of observer- based cosmesis scales employed to evaluate patients treated conservatively for breast cancer," International Journal of Radiation Oncology* Biology* Physics, vol. 11, no. 6, pp. 1117-1119, 1985.

2. J. C. Lowery, E. G. Wilkins, W. M. Kuzon, and J. A. Davis, "Evaluations of aesthetic results in breast reconstruction: an analysis of reliability," Annals of plastic surgery, vol. 36, no. 6, pp. 601-607, 1996.

3. K. Sneeuw, N. Aaronson, J. Yarnold, M. Broderick, J. Regan, G. Ross, and A. Goddard, "Cos- metic and functional outcomes of breast conserving treatment for early stage breast cancer. 1. comparison of patients' ratings, observers' ratings and objective assessments," Radiotherapy and oncology, vol. 25, no. 3, pp. 153-159, 1992. 
4. C. Vrieling, L. Collette, E. Bartelink, J. H. Borger, S. J. Brenninkmeyer, J.-C. Horiot, M. Pier- art, P. M. Poortmans, H. Struikmans, E. Van der Schueren et al., "Validation of the methods of cosmetic assessment after breast-conserving therapy in the eortc boost versus no boost trial," International Journal of Radiation Oncology* Biology* Physics, vol. 45, no. 3, pp. 667-676, 1999.

5. R. Yavuzer, S. Smirnes, and I. T. Jackson, "Guidelines for standard photography in plastic surgery," Annals of plastic surgery, vol. 46, no. 3, pp. 293-300, 2001.

6. C. P. Honrado and W. F. Larrabee Jr, "Update in three-dimensional imaging in facial plastic surgery," Current opinion in otolaryngology \& head and neck surgery, vol. 12, no. 4, pp. 327-331, 2004.

7. V. F. Ferrario, C. Sforza, C. Dellavia, G. M. Tartaglia, A. Colombo, and A. Caru', "A quantita- tive three-dimensional assessment of soft tissue facial asymmetry of cleft lip and palate adult patients," Journal of Craniofacial Surgery, vol. 14, no. 5, pp. 739-746, 2003.

8. S. Lee, "Three-dimensional photography and its application to facial plastic surgery," Archives of Facial Plastic Surgery, vol. 6, no. 6, pp. 410-414, 2004.

9. P. Regnault, "Breast ptosis. definition and treatment." Clinics in plastic surgery, vol. 3, no. 2, p. 193, 1976.

10. G. S. LaTrenta and L. A. Hoffman, "Breast reduction," Aesthetic plastic surgery. WB Saunders, Philadelphia, pp. 932-933, 1994.

11. M. S. Kim, G. P. Reece, E. K. Beahm, M. J. Miller, and M. K. Neely Atkinson, E andMarkey, "Objective assessment of aesthetic outcomes of breast cancer treatment: measuring ptosis from clinical photographs," Computers in Biology and Medicine, vol. 37, no. 1, pp. 49-59, 2007.

12. M. Kawale, "Automated identification of fiducial points on three-dimensional torso images," M.S. thesis, Dept. Comp.Sc., University of Houston, Houston, TX, 2010.

13. J. Lewis Jr, "Mammary ptosis," Aesthetic Breast Surgery. Baltimore, Williams \& Wilkins, pp. 130145, 1983.

14. R. R. Brink, "Evaluating breast parenchymal maldistribution with regard to mastopexy and augmentation mammaplasty," Plastic and reconstructive surgery, vol. 86, no. 4, pp. 715-719, 1990.

15. R. R. Brink, "Management of true ptosis of the breast," Plastic and reconstructive surgery, vol. 91, no. 4, pp. 657-662, 1993.

16. L. Kirwan, "A classification and algorithm for treatment of breast ptosis," Aesthetic Surgery Journal, vol. 22, no. 4, pp. 355-363, 2002.

17. V. L. de la Torre J, "Breast mastopexy," 2007. [Online]. Available: http://www.emedicine.com/plastic/topic128.htm.

18. M. A. Shiffman, "Classification of breast ptosis," in Breast Augmentation, M. A. Shiffman, Ed. Springer Berlin Heidelberg, 2009, pp. 251-255. [Online]. Available: http://dx.doi.org/10.1007/978-3540-78948-2 31.

19. A. Bose, S. Shah, G. Reece, M. Crosby, E. Beahm, M. Fingeret, M. Markey, and F. Merchant, "Automated spatial alignment of $3 d$ torso images," in Engineering in Medicine and Biology Society,EMBC, 2011 Annual International Conference of the IEEE, 2011, pp. 8455-8458.

20. J. J. Koenderink and A. J. van Doorn, "Surface shape and curvature scales," Image and vision computing, vol. 10, no. 8, pp. 557-564, 1992.

21. D. Cohen-Steiner and J.-M. Morvan, "Restricted delaunay triangulations and normal cycle," in Proceedings of the nineteenth annual symposium on Computational geometry. ACM, 2003, pp. 312-321.

22. S.-H. Cha and S. N. Srihari, "On measuring the distance between histograms," Pattern Recognition, vol. 35, no. 6, pp. 1355-1370, 2002. 